\title{
Longitudinal Microvascular Changes on Optical Coherence Tomographic Angiography after Macula-Off Rhegmatogenous Retinal Detachment Repair Surgery
}

\author{
Sang Hyu Nam ${ }^{\mathrm{a}}$ Kiyoung Kim ${ }^{\mathrm{a}}$ Eung Suk Kim ${ }^{\mathrm{a}}$ Do Gyun Kim ${ }^{\mathrm{b}}$ \\ Seung-Young $\mathrm{Yu}^{\mathrm{a}}$ \\ a Department of Ophthalmology, Kyung Hee University Hospital, Kyung Hee University, \\ Seoul, Republic of Korea; ${ }^{b}$ Department of Ophthalmology, Hanyang University College of Medicine \\ Myongji Hospital, Goyang, Republic of Korea
}

\section{Keywords}

Rhegmatogenous retinal detachment surgery .

Swept-source optical coherence tomographic

angiography · Retinal microstructure

\begin{abstract}
Purpose: To investigate longitudinal changes and associated factors for microvascular parameters in macula-off rhegmatogenous retinal detachment (RRD) after repair surgery. Methods: This is a retrospective study of 34 eyes with macula-off RRD. The foveal avascular zone (FAZ) area and parafoveal vessel density were recorded every 3 months for 1 year after surgery. We analyzed $3 \times 3 \mathrm{~mm}$ and $6 \times 6 \mathrm{~mm}$ sweptsource optical coherence tomographic (OCT) angiography images to quantify microvascular parameters. Individual clinical factors and pre- and postoperative OCT images were analyzed to find factors associated with changes in the FAZ area and vessel density 12 months after surgery. Results: The differences in FAZ area and vessel density between the operated and fellow eye at 3, 6, 9, and 12 months postoperatively were $0.127 \pm 0.05,0.111 \pm 0.06,0.108 \pm 0.07$, and $0.105 \pm$ $0.06 \mathrm{~mm}^{2}$ and $4.33 \pm 1.42,3.56 \pm 2.05,2.73 \pm 1.72$, and 2.61 $\pm 1.71 / \mathrm{mm}$. Preoperative sensory retina thickness and surgi-
\end{abstract}

karger@karger.com

(c) 2020 S. Karger AG, Basel

www.karger.com/oph

Karger cal methods significantly correlated with decreased vessel density at 12 months. Surgical method-based analysis found that postoperative vessel density damage in the pars plana vitrectomy (PPV) group was more prominent than in the buckling group. Conclusion: After macula-off RRD surgery, microvascular parameters were impaired after successful anatomical repair but recovered over 12 months. The PPV group exhibited significantly lower postoperative parafoveal vessel density than the buckling group.

(c) 2020 S. Karger AG, Basel

\section{Introduction}

Rhegmatogenous retinal detachment (RRD) is a vision-threatening disease caused by a retinal break, allowing vitreous fluid into the subretinal space. Consequently, the sensory retina detaches from the retinal pigment epithelium (RPE). Although successful retinal reattachment has been reported in up to $90 \%$ using either scleral buck-

S.H.N. and K.K. contributed equally to this paper. 
ling or pars plana vitrectomy (PPV), anatomical success does not necessarily have good visual outcomes [1]. Several attempts have been made to predict postoperative visual outcomes in patients with macula-off RRD. Previous studies used spectral domain optical coherence tomography (SD-OCT) to evaluate retinal changes following RRD repair in an effort to understand prognostic factors related to surgical and visual outcomes [2,3]. Regarding microstructure on SD-OCT, undulation of the outer retina, subretinal fluid (SRF) height, and backreflection integrity have been suggested as prognostic factors $[4,5]$. Undulation of the separated outer retina was associated with visual outcomes, and SRF height has been suggested as a prognostic factor for vision because it can affect the interaction between the neurosensory retina and RPE $[6$, 7].

The foveal avascular zone (FAZ) is a specialized region in the human retina responsible for central vision with the highest cone photoreceptor density [8]. FAZ area and visual acuity (VA) are correlated in several retinal vascular diseases [9]. Moreover, various retinal microvasculature-related parameters, such as FAZ area, FAZ circularity, vessel density, and perfusion density correlate with visual function in the progression of retinal disease [10, 11]. Optical coherence tomography angiography (OCTA) is a newly developed noninvasive method for visualizing retinal microcirculation [12]. OCTA can also reproducibly measure the dimensions of the FAZ and provide quantitative microvascular information $[13,14]$. Previous studies showed a relationship between RRD and vascular changes in the macula, including the central retinal artery, short posterior ciliary artery, and flow to the optic nerve head [15]. However, so far few studies examined long-term changes in microvascular structure and correlated clinical factors, especially after vitrectomy and scleral buckling surgery. Therefore, our study investigated changes in microvascular parameters on OCTA, such as FAZ and vessel density, following successful macula-off RRD repair and identified prognostic factors associated with these changes.

\section{Methods}

\section{Study Subjects}

We analyzed 34 eyes in 34 patients diagnosed with macula-off RRD who underwent uncomplicated surgery between April 1, 2016, and November 1, 2018, at the Department of Ophthalmology, Kyung Hee University Hospital. This study was reviewed and approved by the Institutional Review Board of Kyung Hee University Hospital and conducted in accordance with the tenets of the Declaration of Helsinki.

Microvascular Changes after Macula-Off RRD Surgery
Inclusion criteria comprised nontraumatic macula-off RRD successfully repaired following surgery and a minimum 12-month follow-up after surgery. Patients with other ocular diseases, such as diabetic retinopathy or diabetic macular edema, were excluded. All patients underwent baseline ophthalmic examination including best-corrected VA (BCVA), slit lamp examination, color fundus photography, ultrasonography, SD-OCT, and swept-source OCTA (SS-OCTA). Evaluations were repeated every 3 months after surgery. BCVA was converted to the logarithm of minimum angle of resolution $(\log M A R)$ for statistical analyses. Measurements made in the unaffected fellow eye were used as control data.

\section{Surgical Procedures for RRD Surgery}

All patients underwent PPV or scleral buckling, with/without encircling. PPV was performed using a 23-gauge system (Constellation ${ }^{\circledR}$ Vision System, Alcon Laboratories Inc., Fort Worth, TX, USA) with complete fluid-air exchange and air-gas $\left(\mathrm{SF}_{6}\right)$ exchange. Cataract surgery with phacoemulsification and intraocular lens implantation was concomitantly performed if the cataract was visually significant or it was deemed necessary. During scleral buckling, a segmented silicone sponge, with/without an encircling band, was located on the sclera. Cryotherapy was performed around the retinal tear. External SRF drainage was performed at the surgeon's discretion. Intraocular tamponade was performed using gas $\left(\mathrm{SF}_{6}\right)$.

\section{SD-OCT and SS-OCTA Imaging}

Pre- and postoperative OCT images were analyzed using a Spectralis OCT (Heidelberg Engineering, Heidelberg, Germany). Preoperative OCT analysis included backreflection, foveal SRF height, separated outer retina undulation, foveal sensory retinal thickness, and intraretinal cystic cavities in the inner nuclear layer or outer nuclear layer, as depicted in Figure 1a. Backreflection was defined as the integrity of the smudged photoreceptor inner/outer segment (IS/OS) layer line. Dropouts of backreflection were seen from the photoreceptor IS/OS layer line to beneath and in contact with the external limiting membrane (ELM) meaning defects of the foveal photoreceptor IS/OS layer [2]. Foveal SRF height was measured manually using a caliper in the Heidelberg Retina Angiography system (HRA-2, Heidelberg Engineering) between the inner border of the RPE and outer border of the backreflection. Foveal sensory retinal thickness was also measured manually, from the inner border of the RPE to the internal limiting membrane. Postoperative OCT values included the status of the photoreceptor IS/OS junction or ellipsoid zone, ELM integrity, intraretinal cystic cavities in the inner and/or outer nuclear layer, presence of residual SRF, foveal sensory retinal thickness, and macular ganglion cell-inner plexiform layer thickness.

FAZ measurements were performed using an SS-OCTA system (ELITE 9000, Carl Zeiss Meditec, Dublin, OH, USA). All OCTA imaging was performed by acquiring $3 \times 3 \mathrm{~mm}$ and $6 \times 6 \mathrm{~mm}$ macular cubes. En face angiography images of the retinal vasculature were generated of the superficial capillary plexus (SCP) based on automated layer segmentation performed by OCTA system software. The FAZ area was defined as the area inside the central border of the capillary network. The boundary of the FAZ on $3 \times$ $3 \mathrm{~mm}$ angiography images of the SCP layer was manually delineated by 2 experts independently. The FAZ area was automatically calculated using ImageJ (National Institutes of Health, Bethesda, MD, USA; Fig. 1b, c). Vessel density was measured as the percent-

Ophthalmologica 2021;244:34-41 

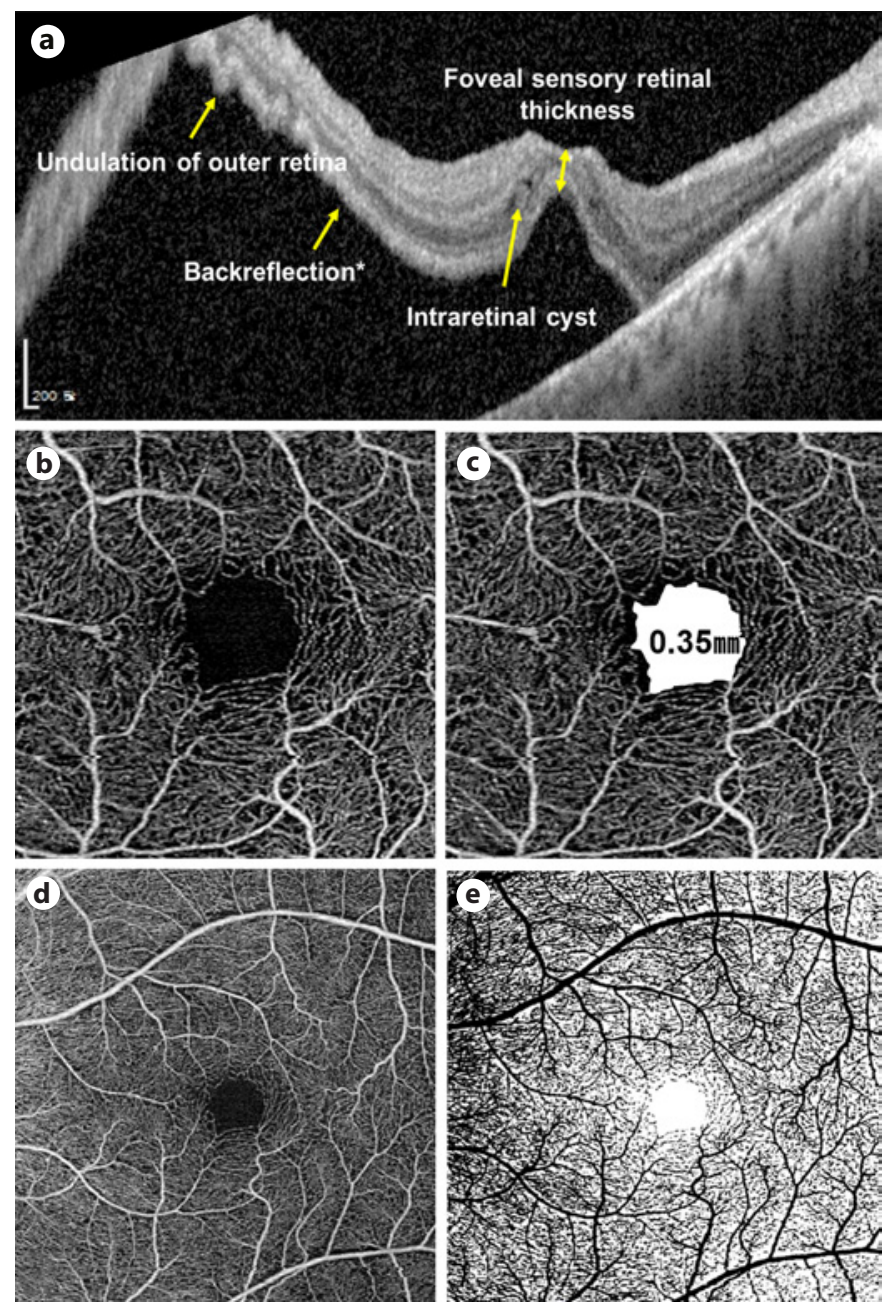

Fig. 1. a Cross-sectional optical coherence tomography images through the fovea. The foveal sensory retinal thickness was measured manually, from the inner border of the retinal pigment epithelium (RPE) and the inner border of the internal limiting membrane. Backreflection $\left(^{*}\right)$ was defined as a smudged external limiting membrane and ellipsoid zone. The height of the subretinal fluid at the fovea was measured between the inner border of the RPE and outer border of the backreflection. $\mathbf{b}$ Illustration of the quantification of microvascular parameters. $3 \times 3 \mathrm{~mm}$ sweptsource optical coherence tomography angiography (SS-OCTA) en face image of the superficial capillary plexus (SCP) layer. c Manual delineation of the foveal avascular zone (FAZ) area outline by experts. The FAZ area was then automatically calculated using ImageJ. d $6 \times 6 \mathrm{~mm}$ SS-OCTA en face image of the SCP layer. e The vessel density was calculated using ImageJ.

age of the total area occupied by blood vessels within the $6 \times 6 \mathrm{~mm}$ images of the SCP layer. Images were transformed into binary images by using Image J and applying a mean threshold algorithm; vessel density was automatically generated (Fig. 1d, e). All OCTA scans were reviewed by 2 retina specialists (K.K. and S.Y.Y.) to ensure quality.
Table 1. Subject baseline demographic and ocular characteristics of 34 patients with macula-off RRD

\begin{tabular}{ll}
\hline Characteristics & Mean \pm SD \\
\hline Eyes, $n$ & 34 \\
Age, years & $48.1 \pm 17.8$ \\
Male:female ratio & $20: 14$ \\
Duration of symptoms, week & $2.1 \pm 2.6$ \\
Axial length, mm & $25.9 \pm 1.7$ \\
Refractive errors, dpt & $-2.87 \pm 3.25$ \\
Phakic:pseudophakic eyes, $n$ & $32: 2$ \\
BCVA at baseline, logMAR & $0.73 \pm 0.38$ \\
Foveal sensory retinal thickness, $\mu \mathrm{m}$ & $243.5 \pm 104$ \\
Surgical methods, $n$ (\%) & \\
$\quad$ Scleral buckling & $18(52.9)$ \\
Scleral buckling and encircling & $7(20.7)$ \\
$\quad$ Pars plana vitrectomy & $9(26.4)$
\end{tabular}

$\mathrm{RRD}$, rhegmatogenous retinal detachment; $\mathrm{SD}$, standard deviation; BCVA, best-corrected visual acuity; logMAR, logarithm of the minimum angle of resolution.

\section{Statistical Analysis}

Patient characteristics were retrieved from medical charts. Repeated-measures analysis of variance was used to compare baseline BCVA and central foveal thickness at each follow-up visit. The Kruskal-Wallis and Mann-Whitney U tests were used to compare mean BCVA and central foveal thickness between the sclera buckling and PPV groups. Spearman's rank correlation analysis was used for univariate analysis to identify significant visual predictive factors, followed by stepwise multiple regression analysis. All statistical analyses were performed using SPSS (version 18 for Windows, SPSS Inc., Chicago, IL, USA). Statistical significance was defined as $p<0.05$.

\section{Results}

\section{Baseline Characteristics}

Thirty-four eyes in 34 patients (20 male, 14 female) with macula-off RRD were included in this study. The mean age was $48.1 \pm 17.8$ years, and the mean duration of macula-off RRD before surgery was $2.1 \pm 2.6$ weeks. Baseline subject demographic parameters and ocular characteristics are summarized in Table 1. Preoperative BCVA ( $\log \mathrm{MAR})$ was $0.73 \pm 0.38$, and the mean axial length was $25.9 \pm 1.7 \mathrm{~mm}$. At the time of retinal detachment surgery, 32 patients were phakic (94.1\%), and 2 patients (5.9\%) were pseudophakic; 25 patients underwent scleral buckling with/without combined encircling (73.6\%), and 9 patients underwent PPV (26.4\%).
36

Ophthalmologica 2021;244:34-41

DOI: $10.1159 / 000508464$
$\mathrm{Nam} / \mathrm{Kim} / \mathrm{Kim} / \mathrm{Kim} / \mathrm{Yu}$ 
Table 2. Factors associated with FAZ area change after 12 months of surgery

\begin{tabular}{|c|c|c|c|c|}
\hline & \multicolumn{2}{|c|}{ Univariate analysis } & \multicolumn{2}{|c|}{ Multivariate analysis } \\
\hline & $r$ & $p$ value & $\beta$ & $p$ value \\
\hline \multicolumn{5}{|l|}{ Clinical factors } \\
\hline Age & 0.072 & 0.739 & 0.080 & 0.709 \\
\hline Duration of $S \mathrm{x}$ & 0.050 & 0.815 & 0.098 & 0.648 \\
\hline Baseline BCVA (logMAR) & -0.315 & 0.133 & -0.116 & 0.588 \\
\hline Axial length & -0.070 & 0.744 & -0.069 & 0.749 \\
\hline Presence of DM & -0.218 & 0.307 & -0.123 & 0.566 \\
\hline Surgical method & -0.035 & 0.872 & -0.203 & 0.341 \\
\hline \multicolumn{5}{|l|}{ Preop. OCT factors } \\
\hline Intraretinal cyst & 0.026 & 0.906 & -0.036 & 0.869 \\
\hline Undulation of outer retina & -0.037 & 0.863 & -0.171 & 0.424 \\
\hline Backreflection integrity & 0.037 & 0.863 & -0.063 & 0.770 \\
\hline Foveal sensory retina thickness & -0.478 & $0.015^{*}$ & -0.338 & 0.106 \\
\hline SRF height & -0.194 & 0.363 & -0.332 & 0.113 \\
\hline \multicolumn{5}{|l|}{ Postop. OCT factors } \\
\hline IS/OS integrity & 0.037 & 0.863 & -0.063 & 0.770 \\
\hline Residual SRF & 0.006 & 0.976 & -0.135 & 0.531 \\
\hline mGCIPL thickness & 0.055 & 0.797 & -0.077 & 0.721 \\
\hline
\end{tabular}

* $p$ value $<0.05$ was set for clinical significance in univariate regression. FAZ, foveal avascular zone; BCVA, best-corrected visual acuity; Sx, symptom; logMAR, logarithm of the minimum angle of resolution; DM, diabetes mellitus; OCT, optical coherence tomography; SRF, subretinal fluid; IS/OS, inner segment/outer segment; mGCIPL, macular ganglion cell inner plexiform layer.

\section{Changes in BCVA}

The mean BCVA at baseline was $0.78 \pm 0.38 \log \mathrm{MAR}$, improving to $0.21 \pm 0.25 \log \mathrm{MAR}$ at 12 months after surgery $(p<0.05)$. The mean BCVA was significantly improved at all follow-up time points $(p<0.05)$. Subgroup analysis showed that the mean BCVA was improved following both PPV (PPV group) and scleral buckling, with/ without combined encircling, a year after surgery $(p<$ 0.05).

Univariate analysis identified several factors significantly associated with postoperative BCVA at 12 months after surgery. Preoperative VA $(p=0.002)$, symptom duration $(p=0.031)$, preoperative backreflection integrity $(p=0.006)$, SRF height on OCT $(p=0.007)$, postoperative IS/OS integrity $(p=0.006)$, and residual SRF $(p=0.002)$ were associated with postoperative BCVA at 12 months. However, in the multivariate analysis, only baseline BCVA and symptom duration were significantly associated with final BCVA $(p<0.001, p=0.046$, respectively).

\section{Pre- and Postoperative Findings on SD-OCT}

The mean foveal sensory retinal thickness at baseline was $243.5 \pm 104.0 \mu \mathrm{m}$. The mean SRF height was $489.3 \pm$

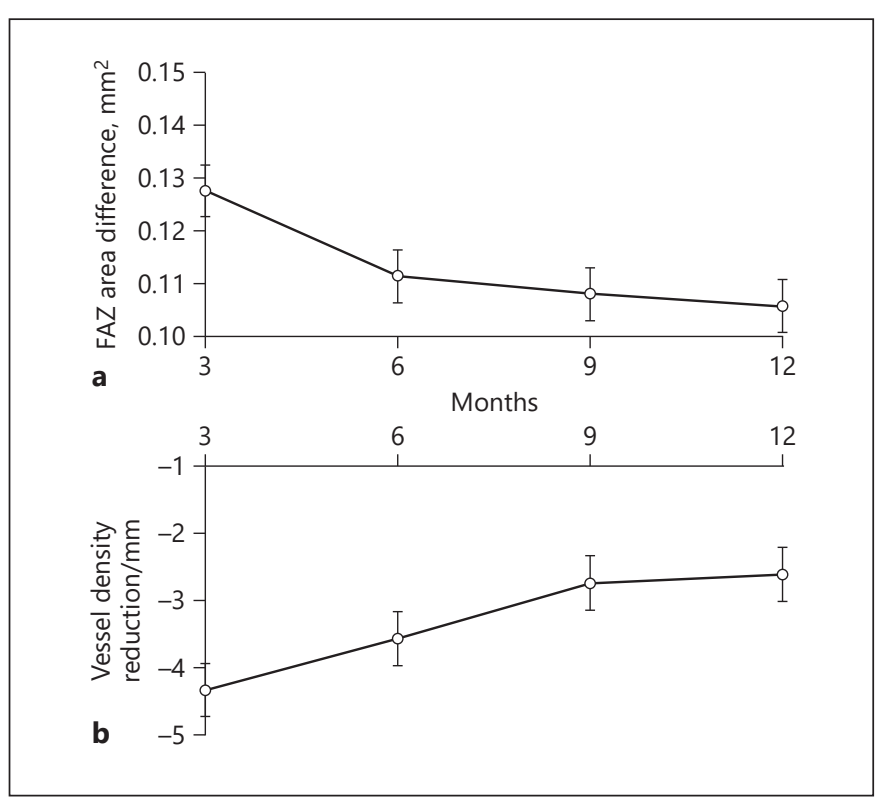

Fig. 2. Changes in the mean foveal avascular zone (FAZ) area and parafoveal vessel density difference (relative to the contralateral eye) of patients after surgery. a The mean FAZ area difference was significantly reduced during the 12 months after surgery $(p<0.05)$. b The mean vessel density recovered 12 months after surgery. 
$367.1 \mu \mathrm{m}$. Twenty-seven patients $(79.4 \%)$ had intraretinal cystic cavities. Undulation of the separated outer retina was observed in 24 patients (70.6\%). A dropout of backreflection in the fovea was observed in 12 patients (35.3\%). The IS/OS junction (ellipsoid zone) was disrupted in 14 patients (41.1\%) at 3 months postoperatively. These patients still showed disruption of the photoreceptor IS/OS junction (ellipsoid zone) on OCT 12 months after surgery. After retinal reattachment surgery, residual SRF at the fovea was detected in 11 patients $(32.3 \%)$ using SDOCT at 3 months (8 patients with scleral buckling with/ without combined encircling, 3 patients with PPV). All patients showed complete resolution of the SRF at 1 year. The mean macular ganglion cell-inner plexiform layer thickness was $73.9 \pm 13.5 \mu \mathrm{m}$ at 3 months and $74.3 \pm 12.7$ $\mu \mathrm{m}$ at 12 months.

\section{Changes in FAZ Area and Vessel Density Difference}

The FAZ area of the SCP layer at 3 months postoperatively was greater in RRD eyes than in the contralateral eye. The mean difference in FAZ area at each subsequent follow-up visit was significantly lower than that at 3 months. The FAZ area difference after surgery at each follow-up visit was $0.127 \pm 0.05,0.111 \pm 0.06,0.108 \pm 0.07$, and $0.105 \pm 0.06 \mathrm{~mm}^{2}$ at $3,6,9$, and 12 months, respectively (Fig. 2a). Parafoveal vessel density of the SCP layer was significantly lower at 3 months in RRD eyes than in the contralateral eye. The vessel density difference after surgery at each follow-up visit was $-4.33 \pm 1.42,-3.56 \pm$ $2.05,-2.73 \pm 1.72$, and $-2.61 \pm 1.74 / \mathrm{mm}$ at $3,6,9$, and 12 months, respectively. The decrease in vessel density in the repaired eyes progressively recovered over 12 months (Fig. 2b). The correlations between BCVA and microvascular OCTA parameters were analyzed. Neither the FAZ area nor parafoveal vessel density of the SCP significantly correlated with postoperative VA.

\section{Factors Associated with Difference in FAZ Area and Vessel Density}

Baseline characteristics, and pre- and postoperative SD-OCT factors were analyzed to find factors that correlated with change in FAZ area and vessel density at 12 months postoperatively. Preoperative foveal sensory retina thickness significantly correlated in the univariate analysis $(p=0.015)$ but not in the multivariate analysis (Table 2). The vessel density difference at 12 months was significantly associated with surgical method and foveal sensory retina thickness in both univariate analysis $(p=$ $0.039,0.028$, respectively) and multivariate analysis $(p=$ $0.011,0.012$, respectively; Table 3 ).
Table 3. Factors associated with vessel density difference after 12 months of surgery

\begin{tabular}{|c|c|c|c|c|}
\hline & \multicolumn{2}{|c|}{$\begin{array}{l}\text { Univariate } \\
\text { analysis }\end{array}$} & \multicolumn{2}{|c|}{$\begin{array}{l}\text { Multivariate } \\
\text { analysis }\end{array}$} \\
\hline & $r$ & $p$ value & $\beta$ & $p$ value \\
\hline \multicolumn{5}{|l|}{ Clinical factors } \\
\hline Age & -0.048 & 0.911 & 0.274 & 0.107 \\
\hline Duration of Sx, weeks & -0.485 & 0.223 & 0.041 & 0.862 \\
\hline Baseline BCVA (logMAR) & 0.240 & 0.568 & -0.064 & 0.818 \\
\hline Axial length & 0.048 & 0.911 & -0.224 & 0.226 \\
\hline Presence of DM & -0.412 & 0.310 & 0.033 & 0.873 \\
\hline Surgical method & 0.732 & $0.039^{*}$ & 0.616 & $0.011^{\dagger}$ \\
\hline \multicolumn{5}{|l|}{ Preop. OCT factors } \\
\hline Intraretinal cyst & 0.169 & 0.689 & 0.050 & 0.788 \\
\hline Undulation of outer retina & 0.056 & 0.895 & -0.262 & 0.090 \\
\hline Backreflection integrity & 0.056 & 0.895 & -0.262 & 0.090 \\
\hline \multicolumn{5}{|l|}{ Foveal sensory retina } \\
\hline thickness & 0.762 & $0.028^{*}$ & 0.608 & $0.012^{\dagger}$ \\
\hline SRF height & 0.238 & 0.570 & -0.272 & 0.130 \\
\hline \multicolumn{5}{|l|}{ Postop. OCT factors } \\
\hline IS/OS integrity & 0.056 & 0.895 & -0.262 & 0.090 \\
\hline Residual SRF & 0.169 & 0.689 & 0.050 & 0.788 \\
\hline mGCIPL thickness & 0.144 & 0.734 & 0.042 & 0.839 \\
\hline
\end{tabular}

$* p$ value $<0.05$ was set for clinical significance in univariate regression. ${ }^{\dagger} p$ value $<0.05$ was set for clinical significance in multivariate regression. BCVA, best-corrected visual acuity; Sx, symptom; logMAR, logarithm of the minimum angle of resolution; DM, diabetes mellitus; OCT, optical coherence tomography; SRF, subretinal fluid; IS/OS, inner segment/outer segment; mGCIPL, macular ganglion cell inner plexiform layer.

\section{Subgroup Analysis by Surgical Methods}

The vessel density difference from that in the control eye 3 months after surgery was $-3.74 \pm 1.79$ and $-5.30 \pm$ $1.11 / \mathrm{mm}$ in the scleral buckling and PPV groups, respectively. After 6 months, the differences were $-2.44 \pm 1.27$ and $-5.43 \pm 1.62 / \mathrm{mm}$, after 9 months $-1.92 \pm 1.38$ and $-4.1 \pm 0.26 / \mathrm{mm}$, and after 12 months $-1.7 \pm 1.21$ and $-4.13 \pm 0.21 / \mathrm{mm}$, respectively (Fig. 3 ). The difference in vessel density reduction between the 2 surgical groups was significant at every follow-up time point. Postoperative vessel density damage was greater and recovery slower in the PPV group.

\section{Discussion}

We evaluated serial changes in microvascular parameters and various factors associated with postoperative visual outcome after macula-off RRD surgery, including
38

Ophthalmologica 2021;244:34-41 DOI: $10.1159 / 000508464$
$\mathrm{Nam} / \mathrm{Kim} / \mathrm{Kim} / \mathrm{Kim} / \mathrm{Yu}$ 


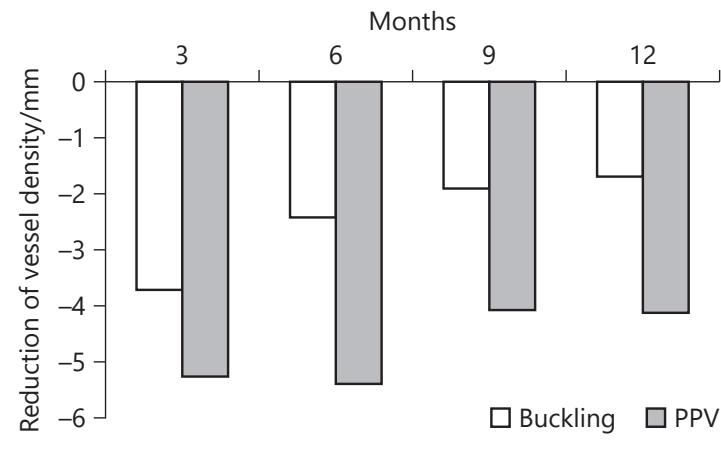

Fig. 3. Serial changes in vessel density reduction from baseline until 12 months after surgery performed using different surgical methods. Postoperative reduction in vessel density was significantly prominent in the vitrectomy group relative to that in the scleral buckling group during 12 months of follow-up.

clinical, surgical, and SD-OCT factors. Although many factors were identified to predict postoperative visual acuity in eyes with macula-off RRD, preoperative BCVA was suggested to be the most important predictor of postoperative visual recovery after macula-off RRD surgery [16]. In our univariate analysis, baseline BCVA, symptom duration, preoperative SD-OCT backreflection integrity, SRF height, postoperative SD-OCT IS/OS integrity, and residual SRF were significantly correlated with final BCVA. The integrity of the photoreceptor and ELM are prognostic factors for visual function in various diseases, including age-related macular degeneration, diabetic macular edema, and retinal vein occlusion. Backreflection reflects the integrity of the photoreceptor layer in macula-off RRD patients; in the present study, the integrity of backreflection on baseline OCT was the only prognostic factor for postoperative visual outcome in maculaoff RRD patients. Previous studies found that the postoperative integrity of the ELM and photoreceptor IS/OS junction was disrupted in RRD patients with poor visual outcomes after successful surgery [17]. The backreflection status on preoperative SD-OCT may be a useful predictive factor for postoperative visual outcomes in macula-off RRD patients, because the photoreceptor IS/OS junction and ELM integrities of RRD patients are difficult to identify. Earlier studies reported that duration of retinal detachment correlated with visual recovery [18]. Within 7 days of surgery, BCVA was significantly better in those with shorter symptom duration rather than the group with longer duration. The length of macular de- tachment within the first week does not significantly influence VA after scleral buckling surgery [19]. Our study found a significant correlation between retinal detachment duration and final BCVA. This is because photoreceptor cell apoptosis progresses slowly in macula-off RRD eyes.

Changes in the FAZ area can indicate alterations in the microcirculation state of the fovea. Enlargement of the FAZ can be seen in ischemic diseases and has been shown to be associated with poor visual outcomes [20]. Recently, Woo et al. [21] reported that superficial and deep FAZ areas were larger in eyes with macula-off RRD after vitrectomy than fellow eyes. We also found that the FAZ area of eyes following RRD repair surgery was significantly larger than that of the control eye. In terms of vessel density, Agarwal et al. [22] found that RRD eyes successfully treated with PPV had a lower vessel density in the superficial and deep retinal plexuses than healthy eyes. Our results showed that macular vessel density decreased from 3 months after RRD repair surgery relative to that in control eyes. This may be because fluid passes through into the subretinal space in the retinal tear. The detached retina becomes hypoxic, and diffuse vascular occlusions can be observed. Consequently, retinal capillary dilatation and hyperpermeability were observed in the detached retina, and levels of inflammatory mediators, like prostaglandins and cytokines, are elevated in the SRF, increasing vascular permeability $[23,24]$. Additionally, we found that the FAZ area and vessel density through the 12 months after surgery showed progressive recovery, suggesting that progressive recovery of microvascular parameters might be associated with the recovery of attached retinal layer integrity.

Even though the macula was well attached after repair, functional recovery of the macula may not have been entirely complete. We suspect that restoration of blood perfusion may take longer. Few studies have investigated the relationship between postoperative BCVA and OCTA parameters in eyes with macula-off. Woo et al. [21] showed that final postoperative BCVA at 2 months negatively correlated with both the superficial and deep FAZ area, while Sato et al. [25] performed a follow-up study and found no correlation between BCVA and FAZ area 6 months postoperatively. We found the loss of the significant correlation between postoperative BCVA and change in FAZ area or vessel density at the 12-month follow-up. This discrepancy may be due to the variability of the FAZ area and vessel density dependent on the OCTA segmentation in individuals. Different quantification methods for OCTA parameters and longer follow-up period might 
be possible reasons for the inconsistent results. Binarization data show that there was a significant decrease in vessel flow density in the inferior sector of the peripapillary area 3 months after PPV with internal limiting membrane peeling. According to Rabiolo et al. [26], the FAZ area in all plexuses is very reliable, even if calculated based on cube scans of different sizes. However, reliability is higher when measured using smaller scans. Vessel density is also highly reliable, and the results depend on the cube size. Conversely, vessel density results depend on the angiocube size, although their interrater reliability is extremely high. Studies involving OCTA should take into consideration that scan size may influence macular perfusion parameters and interrater reliability. Therefore, further associations between visual recovery and retinal perfusion after successful repair of the RRD should be identified.

In multiple regression analysis, we found 2 factors significantly associated with final vessel density difference at 12 months, foveal sensory retinal thickness and surgical methods. Previously, Dell'Omo et al. [27] reported progressive recovery of central foveal thickness to a level similar to that of the fellow eye at 12 months after macula-off RRD surgery. The progressive increase in central retinal thickness was accompanied by restoration of outer retinal band integrity and postoperative BCVA, suggesting that central retinal thickness might be a biomarker for the recovery of retinal microstructure.

Few studies have evaluated the effects of PPV or scleral buckling on macular perfusion. Sato et al. [25] reported that ocular microcirculation is normal 6 months after scleral buckling or vitrectomy for RRD. Iwase et al. [28] reported that retinal blood flow to the optic nerve head is significantly reduced in eyes with RRD preoperatively but can recover 6 months after successful RRD repair with PPV. Furthermore, scleral buckling has been reported to potentially reduce retinal and choroidal blood flow due to direct obstruction of choroidal venous drainage. Tsen et al. [29] have recently compared retinal microvascular changes in macula-off RRD using OCTA between different surgical treatments. They reported that the combination of PPV and scleral buckling resulted in lower vessel density in the SCP and deep capillary plexus than buckling or PPV alone. Our data demonstrate a vessel density difference between the 2 surgical groups. Both groups showed vessel density recovery 12 months after surgery, but eyes with PPV had significantly lower vessel density than the scleral buckling group at each follow-up time point. The reduced vessel density in the PPV group might be because uncomplicated PPV can damage the superfi- cial microstructure of retinal vessels during surgery. Furthermore, the PPV group might have a relatively chronic or complicated condition, delaying recovery of retinal perfusion after attachment.

Our study had several limitations. First, it used a retrospective design with a relatively small sample size. Second, the FAZ area and vessel density were measured in only the SCP slab. Current OCTA technology cannot remove all projection artifacts at the level of the deep capillary plexus. Thus, vessels in the SCP may have been included in the analysis of the deep capillary plexus structure, causing inconsistent quantification of the FAZ area and vessel density. Large prospective studies will provide data to better understand changes in vascular parameters and their relationship with functional and anatomical results.

In conclusion, BCVA improved significantly following successful retinal reattachment surgery. The FAZ area increased and vessel density decreased relative to that in the control eye, but progressively recovered over 12 months and demonstrated no correlation with BCVA. Thinner preoperative foveal sensory thickness was associated with lower vessel density in the SCP 12 months postoperatively. Moreover, the PPV group had lower vessel density in the SCP than the scleral buckling group, potentially suggesting PPV could damage the microvascular structure of vessels.

\section{Statement of Ethics}

This study was reviewed and approved by the Institutional Review Board of Kyung Hee University Hospital and conducted in accordance with the tenets of the Declaration of Helsinki.

\section{Disclosure Statement}

The authors have no conflicts of interest to declare.

\section{Funding Sources}

There are no funding sources to declare.

\section{Author Contributions}

All authors were involved in the literature search, drafting, revising, and final approval of the paper.
$\mathrm{Nam} / \mathrm{Kim} / \mathrm{Kim} / \mathrm{Kim} / \mathrm{Yu}$ 


\section{References}

1 Adelman RA, Parnes AJ, Ducournau D; European Vitreo-Retinal Society (EVRS) Retinal Detachment Study Group. Strategy for the management of uncomplicated retinal detachments: the European vitreo-retinal society retinal detachment study report 1 . Ophthalmology. 2013 Sep;120(9):1804-8.

2 Nakanishi H, Hangai M, Unoki N, Sakamoto A, Tsujikawa A, Kita M, et al. Spectral-domain optical coherence tomography imaging of the detached macula in rhegmatogenous retinal detachment. Retina. 2009 Feb;29(2): 232-42.

3 Hirata N, Iwase T, Kobayashi M, Yamamoto $\mathrm{K}, \mathrm{Ra}$ E, Terasaki H. Correlation between preoperative factors and final visual acuity after successful rhegmatogenous retinal reattachment. Sci Rep. 2019 Mar;9(1):3217.

4 Mowatt L, Tarin S, Nair RG, Menon J, Price NJ. Correlation of visual recovery with macular height in macula-off retinal detachments. Eye (Lond). 2010 Feb;24(2):323-7.

5 Ross W, Lavina A, Russell M, Maberley D. The correlation between height of macular detachment and visual outcome in maculaoff retinal detachments of $[\{\mathrm{LT}\}]$ or $=7$ days' duration. Ophthalmology. 2005 Jul;112(7): 1213-7.

6 Hagimura N, Suto K, Iida T, Kishi S. Optical coherence tomography of the neurosensory retina in rhegmatogenous retinal detachment. Am J Ophthalmol. 2000 Feb;129(2): 186-90.

7 Cho M, Witmer MT, Favarone G, Chan RP, D'Amico DJ, Kiss S. Optical coherence tomography predicts visual outcome in maculainvolving rhegmatogenous retinal detachment. Clin Ophthalmol. 2012;6:91-6.

8 Weale RA. Why does the human retina possess a fovea. Nature. 1966 Oct 15;212(5059): 255-6.

9 Arend O, Wolf S, Harris A, Reim M. The relationship of macular microcirculation to visual acuity in diabetic patients. Arch Ophthalmol. 1995 May;113(5):610-4.

10 Dupas B, Minvielle W, Bonnin S, Couturier A, Erginay A, Massin P, et al. Association between vessel density and visual acuity in patients with diabetic retinopathy and poorly controlled type 1 diabetes. JAMA Ophthalmol. 2018 Jul;136(7):721-8.
11 Samara WA, Shahlaee A, Adam MK, Khan MA, Chiang A, Maguire JI, et al. Quantification of diabetic macular ischemia using optical coherence tomography angiography and its relationship with visual acuity. Ophthalmology. 2017 Feb;124(2):235-44.

12 Ferrara D, Waheed NK, Duker JS. Investigating the choriocapillaris and choroidal vasculature with new optical coherence tomography technologies. Prog Retin Eye Res. 2016 May;52:130-55.

13 Mastropasqua R, Toto L, Mastropasqua A, Aloia R, De Nicola C, Mattei PA, et al. Foveal avascular zone area and parafoveal vessel density measurements in different stages of diabetic retinopathy by optical coherence tomography angiography. Int J Ophthalmol. 2017 Oct;10(10):1545-51.

14 Garrity ST, Iafe NA, Phasukkijwatana N, Chen X, Sarraf D. Quantitative analysis of three distinct retinal capillary plexuses in healthy eyes using optical coherence tomography angiography. Invest Ophthalmol Vis Sci. 2017 Oct;58(12):5548-55.

15 Bonfiglio V, Ortisi E, Scollo D, Reibaldi M, Russo A, Pizzo A, et al. Vascular changes after vitrectomy for rhegmatogenous retinal detachment: optical coherence tomography angiography study. Acta Ophthalmol. 2019 Nov; Epub ahead of print.

16 Tani P, Robertson DM, Langworthy A. Prognosis for central vision and anatomic reattachment in rhegmatogenous retinal detachment with macula detached. Am J Ophthalmol. 1981 Nov;92(5):611-20.

17 Kang HM, Lee SC, Lee CS. Association of spectral-domain optical coherence tomography findings with visual outcome of maculaoff rhegmatogenous retinal detachment surgery. Ophthalmologica. 2015;234(2):83-90.

18 Diederen RM, La Heij EC, Kessels AG, Goezinne F, Liem AT, Hendrikse F. Scleral buckling surgery after macula-off retinal detachment: worse visual outcome after more than 6 days. Ophthalmology. 2007 Apr;114 (4):705-9.

19 Ross WH, Kozy DW. Visual recovery in macula-off rhegmatogenous retinal detachments. Ophthalmology. 1998 Nov;105(11):2149-53.
20 Sim DA, Keane PA, Zarranz-Ventura J, Fung S, Powner MB, Platteau E, et al. The effects of macular ischemia on visual acuity in diabetic retinopathy. Invest Ophthalmol Vis Sci. 2013 Mar;54(3):2353-60.

21 Woo JM, Yoon YS, Woo JE, Min JK. Foveal avascular zone area changes analyzed using OCT angiography after successful rhegmatogenous retinal detachment repair. Curr Eye Res. 2018 May;43(5):674-8.

22 Agarwal A, Aggarwal K, Akella M, Agrawal R, Khandelwal N, Bansal R, et al.; OCTA Study Group. Fractal dimension and optical coherence tomography angiography features of the central macula after repair of rhegmatogenous retinal detachments. Retina. 2019 Nov; 39(11):2167-77.

23 Cardillo Piccolino F. Vascular changes in rhegmatogenous retinal detachment. Ophthalmologica. 1983;186(1):17-24.

24 Quintyn JC, Brasseur G. Subretinal fluid in primary rhegmatogenous retinal detachment: physiopathology and composition. Surv Ophthalmol. 2004 Jan-Feb;49(1):96-108.

25 Sato T, Kanai M, Busch C, Wakabayashi T. Foveal avascular zone area after macula-off rhegmatogenous retinal detachment repair: an optical coherence tomography angiography study. Graefes Arch Clin Exp Ophthalmol. 2017 Oct;255(10):2071-2.

26 Rabiolo A, Gelormini F, Marchese A, Cicinelli MV, Triolo G, Sacconi R, et al. Macular perfusion parameters in different angiocube sizes: does the size matter in quantitative optical coherence tomography angiography? Invest Ophthalmol Vis Sci. 2018 Jan;59(1):231-7.

27 dell'Omo R, Viggiano D, Giorgio D, Filippelli M, Di Iorio R, Calo' R, et al. Restoration of foveal thickness and architecture after macula-off retinal detachment repair. Invest Ophthalmol Vis Sci. 2015 Jan;56(2):1040-50.

28 Iwase T, Kobayashi M, Yamamoto K, Yanagida $\mathrm{K}, \mathrm{Ra} \mathrm{E}$, Terasaki $\mathrm{H}$. Changes in blood flow on optic nerve head after vitrectomy for rhegmatogenous retinal detachment. Invest Ophthalmol Vis Sci. 2016 Nov;57(14):622333.

29 Tsen CL, Sheu SJ, Chen SC, Wu TT. Imaging analysis with optical coherence tomography angiography after primary repair of maculaoff rhegmatogenous retinal detachment. Graefes Arch Clin Exp Ophthalmol. 2019 Sep; 257(9):1847-55. 